\title{
Pulse Oximetry Values in Newborns with Critical Congenital Heart Disease upon ICU Admission at Altitude
}

\author{
John S. Kim ${ }^{1, * \mathbb{D}}$, Merlin W. Ariefdjohan ${ }^{2}$, Marci K. Sontag ${ }^{2,3}$ and Christopher M. Rausch ${ }^{1}$ \\ 1 Department of Pediatrics, Heart Institute, Children's Hospital Colorado, \\ University of Colorado School of Medicine, Aurora, CO 80045, USA; \\ Christopher.Rausch@childrenscolorado.org \\ 2 Department of Epidemiology, Colorado School of Public Health, \\ University of Colorado Anschutz Medical Campus, Aurora, CO 80045, USA; \\ Merlin.Ariefdjohan@ucdenver.edu (M.W.A.); msontag@ciinternational.com (M.K.S.) \\ 3 Center for Public Health Innovation at CI International, Littleton, CO 80120, USA \\ * Correspondence: john.kim@childrenscolorado.org; Tel.: +1-720-777-2885
}

Received: 17 September 2018; Accepted: 27 October 2018; Published: 31 October 2018

\begin{abstract}
Pulse oximetry screening for critical congenital heart disease (CCHD) has been recommended by the American Academy of Pediatrics (AAP). The objectives of this study are to describe saturation data, and to evaluate the effectiveness of AAP-recommended pulse oximetry screening guidelines applied retrospectively to a cohort of newborns with known CCHD at moderate altitude (5557 feet, Aurora, Colorado). Data related to seven critical congenital heart disease diagnoses were extracted from electronic health records (pulse oximetry, prostaglandin administration, and oxygen supplementation). Descriptive epidemiologic data were calculated. 158 subjects were included in this analysis; the AAP pulse oximetry screening protocol was applied to 149 subjects. Mean pre-ductal and post-ductal pulse oximetry values of the infants known to have CCHD at $24 \mathrm{~h}$ of life were $87.1 \% \pm 7.2$ and $87.8 \% \pm 6.3$, respectively. Infants treated with prostaglandins and oxygen had lower oximetry readings. The screening algorithm would have identified $80.5 \%$ of infants with known CCHDs (120/149 subjects). Additionally, sequential pulse oximetry screening based on the AAP-recommended protocol was able to identify a true positive screen capture rate of $80.5 \%$ at moderate altitude.
\end{abstract}

Keywords: critical congenital heart disease; pulse oximetry; newborn screening; altitude

\section{Introduction}

Congenital heart disease is among the most common birth defects, with an incidence of approximately 1 per 100 live births. Critical congenital heart disease (CCHD) is defined as a structural heart defect with significant risk for mortality without intensive intervention. CCHD occurs in approximately 4 per 1000 live births, and it is estimated that $13-55 \%$ of newborns with CCHD are discharged from hospital undiagnosed [1-3].

Screening with pulse oximetry has been identified as a low-cost non-invasive test that improves the ability to diagnose CCHD when compared to physical examination alone [4,5]. Several CCHD lesions are particularly amenable to identification via pulse oximetry screening, including truncus arteriosus (TA), transposition of the great arteries (TGA), tricuspid valve atresia (TVA), tetralogy of Fallot (TOF), total anomalous pulmonary venous return (TAPVR), hypoplastic left heart syndrome (HLHS), and pulmonary valve atresia with intact ventricular septum (PA/IVS) [6-8]. The most widely utilized pulse oximetry screening guidelines recommend initiation at or beyond $24 \mathrm{~h}$ of life with 
measurement of pre- and post-ductal saturations (right hand and foot, respectively) [8]. Pulse oximetry screening in the first $24-48 \mathrm{~h}$ after birth has been found to be highly specific (99.9\% specificity; $95 \%$ confidence interval (CI) $99.7,99.9)$, but with a lower sensitivity of $76.3 \%(95 \%$ CI $69.5,82.0)$ in one meta-analysis [9].

The performance of pulse oximetry screening has been evaluated in many studies performed at sea level [10], however, studies at altitude are limited [3,11]. Evaluation of the effectiveness of pulse oximetry screening guidelines is complicated due to the small number of infants likely to be identified through newborn screening as many infants with CCHD are diagnosed prenatally or in the first $24 \mathrm{~h}$ of life, thereby reducing the potential number of infants with undiagnosed CCHD eligible for screening with pulse oximetry. Furthermore, studies of newborn oxygen saturations have suggested a wider range and lower average saturations for infants born at higher altitude, further complicating evaluation of the utility of pulse oximetry in this population [12-15]. We retrospectively reviewed pulse oximetry values for a population of newborns between 24 and $48 \mathrm{~h}$ of life who were known to have CCHD to provide an epidemiologic description of saturation data in newborns with CCHD at altitude.

\section{Materials and Methods}

We performed a retrospective cohort study at a regional tertiary children's hospital at moderate altitude (5557 feet, Aurora, CO, USA). We queried the electronic health record (EHR) for newborns presenting within the first $24-48 \mathrm{~h}$ of life with one of the $7 \mathrm{CCHD}$ diagnoses using International Classification of Disease (ICD) codes in the years 2006-2013 (i2b2; Informatics for Integrating Biology and the Bedside, Version 1.7, Partners HealthCare System, Boston, MA, USA). Subjects included both prenatally and postnatally diagnosed CCHD. We reviewed the EHR for confirmation of diagnosis, pulse oximetry readings, prostaglandin (PGE) administration, and oxygen supplementation data at $24,28,36$, and $48 \mathrm{~h}( \pm 2 \mathrm{~h})$ of life. These time points were within the American Academy of Pediatrics (AAP)-recommended window of screening between 24 and $48 \mathrm{~h}$ [8]. Pulse oximetry was measured continuously in all subjects with simultaneous pre- and post-ductal saturation values recorded in the EHR. Pulse oximetry values were recorded hourly in the EHR via standard clinical practice upon bedside nurse confirmation of the plethysmograph waveform during the patient's usual calm physiologic state.

In a secondary analysis of the pulse oximetry data, we applied a simulated pulse oximetry screening protocol modeled after the recommended AAP algorithm [8]. Pulse oximetry values were categorized based on the AAP screening protocol: (1) passed screen (no concern for CCHD, false negative): saturation $\geq 95 \%$ and $\leq 3 \%$ difference between pre- and post-ductal readings; (2) borderline reading (to be repeated at next time point): saturation $90-94 \%$ and / or $>3 \%$ difference between pre- and post-ductal readings; (3) failed screen (concern for CCHD, true positive): saturation $<90 \%$ in either pre- or post-ductal reading. Up to 3 total hypothetical screens were applied to the retrospective data collected at times closest to 28,36 , and $48 \mathrm{~h}$ of life. Data that resulted in a positive or negative screen for CCHD (based on the AAP protocol) were not evaluated in subsequent time points. Subjects with borderline pulse oximetry values at 3 consecutive readings were considered to meet screening criteria for concern for CCHD. Since all participating subjects had a CCHD diagnosis, subjects in whom pulse oximetry readings raised no concern for CCHD were considered false negative in this hypothetical screening. All other subjects were considered true positives. This secondary analysis allowed us to estimate the general effectiveness of the AAP protocol (i.e., false negative vs. true positive) in a cohort of infants with known CCHD at altitude based on retrospective data.

Study data were collected and maintained by using Research Electronic Data Capture (REDCap) tools hosted at the University of Colorado [16]. This study was approved by the Colorado Multiple Institutional Review Board with a full waiver of Health Insurance and Portability and Accountability Act (HIPAA) authorization. 
Baseline demographic data are presented as means, standard deviations, and range. Group proportions for categorical variables were statistically assessed using the Fisher exact test. The Shapiro-Wilk test for normality was used to evaluate distributions for all continuous variables. Then, the $t$-test and Wilcoxon-Mann-Whitney test were applied to compare continuous variables between groups for those with normal and non-normal distributions, respectively. Pre- and post-ductal pulse oximetry values collected at $24,28,36$, and $48 \mathrm{~h}$ were summarized as a box-whisker plot showing the mean, median, minimum/maximum values, and interquartile ranges. Mean pulse oximetry readings for various types of clinical support (i.e., PGE, oxygen, both, or none) were compared to each other using the Kruskal-Wallis test due to non-normality of data distribution. Post-hoc analysis was made on groups showing statistical difference. Statistical significance level was set at $p<0.05$ and $95 \%$ CI were calculated for mean pulse oximetry values. All analyses were performed using SAS software (version 9.4; SAS Institute, Cary, NC, USA).

\section{Results}

\subsection{Descriptive Analysis of Study Cohort}

Two hundred and fourteen subjects with CCHD were identified. Fifty-six subjects were excluded from data collection due to the following reasons: insufficient pulse oximetry data in the EHR, death prior to $24 \mathrm{~h}$ of life, or atrial septostomy or surgery prior to $24 \mathrm{~h}$ of life. One hundred and fifty-eight subjects were included in this analysis. The distribution of CCHD anatomic diagnoses between the subjects included and excluded from the study was different (Table 1), but we found no other differences between the groups. The mean pre-ductal pulse oximetry readings at $24,28,36$, and $48 \mathrm{~h}$ were $87.1 \pm 7.2 \%$ (95\% CI 85.6, 88.6), $86.7 \pm 7.2 \%(85.2,88.2), 86.8 \pm 7.6 \%(85.2,88.4)$, and $89.3 \pm 6.5 \%(88.0,90.6)$, respectively. The mean post-ductal pulse-oximetry readings were $87.8 \pm 6.3 \%$ (95\% CI 86.6, 89.0), $87.4 \pm 7.3 \%(86.1,88.8), 88.5 \pm 6.7 \%(87.3,89.7)$, and $89.2 \pm 6.3 \%$ $(88.2,90.3)$, respectively. Figure 1 summarizes the overall distribution of pre- and post-ductal saturation readings at the four time points. For descriptive purposes, data were also stratified by diagnoses and pulse oximetry readings for each collection time (Figure 2). To evaluate the effects of treatment on pulse oximetry, we compared pulse oximetry data in infants receiving PGE and oxygen supplementation (Table 2). Newborns treated with both PGE and oxygen had lower pre- and post-ductal saturation at $48 \mathrm{~h}(87.4 \pm 7.3 \%(95 \% \mathrm{CI} 85.0,89.7)$ and $86.3 \pm 7.0 \%(84.3,88.3)$, respectively, $p<0.05)$ when compared to the other treatment categories (PGE only, oxygen only, or no support).

Table 1. Baseline subject characteristics $(n=214)$.

\begin{tabular}{cccc}
\hline Characteristics & Study Subjects $(\boldsymbol{n = 1 5 8 )}$ & Excluded Subjects $(\boldsymbol{n}=\mathbf{5 6})$ & $\boldsymbol{p}$-Value \\
\hline Gestational age, weeks (range) & $38.2 \pm 2.4(29-45)$ & $38.3 \pm 2.4(30-41.3)$ & 0.379 \\
Birth weight, $g$ (range) & $2988.7 \pm 579.3(1230-4165)$ & $3071.0 \pm 671.7(960-4780)$ & 0.277 \\
Male gender, $n(\%)$ & $101(63.9)$ & $35(62.5)$ & 0.849 \\
\hline Race, $n(\%)$ & & & \\
White/Caucasian & $111(70.3)$ & $37(66.1)$ & 0.470 \\
Black/African American & $10(6.3)$ & $3(5.4)$ & \\
Hawaiian/Pacific Islander & $1(0.6)$ & $0(0)$ & $1(1.8)$ \\
Asian & $1(0.6)$ & $1(1.8)$ & \\
American Indian/Alaskan Native & $3(3.2)$ & $9(16.1)$ & 0.088 \\
Other & $26(16.5)$ & $5(8.9)$ & \\
Not specified & $4(2.5)$ & $15(26.8)$ & \\
Ethnicity, $n$ (\%) & & $36(64.3)$ & \\
Hispanic/Latino & $54(34.2)$ & $5(8.9)$ & \\
Not Hispanic/Latino & $100(63.3)$ & & \\
Not specified & $4(2.5)$ & &
\end{tabular}


Table 1. Cont.

\begin{tabular}{|c|c|c|c|}
\hline Characteristics & Study Subjects $(n=158)$ & Excluded Subjects $(n=56)$ & $p$-Value \\
\hline \multicolumn{4}{|l|}{ Apgar score, score (range) } \\
\hline $1 \mathrm{~min}$ & $7 \pm 2(0-9)$ & $7 \pm 2(2-8)$ & 0.409 \\
\hline $5 \mathrm{~min}$ & $8 \pm 1(0-9)$ & $8 \pm 1(4-9)$ & 0.212 \\
\hline Prenatal diagnosis of CCHD, $n(\%)$ & $84(53.2)$ & $20(35.7)$ & 0.152 \\
\hline Family history of CCHD, $n(\%)$ & $18(11.4)$ & $3(5.4)$ & 0.361 \\
\hline \multicolumn{4}{|l|}{ Genetic diagnosis, $n(\%)$} \\
\hline Down syndrome & $2(1.3)$ & $0(0)$ & \multirow{3}{*}{0.825} \\
\hline 22q11 deletion & $6(3.8)$ & $1(1.8)$ & \\
\hline None specified & $150(94.9)$ & $50(89.3)$ & \\
\hline \multicolumn{4}{|l|}{ CCHD diagnosis, $n(\%)$} \\
\hline HLHS & $48(30.4)$ & $17(30.4)$ & \multirow{8}{*}{$0.002 *$} \\
\hline TOF & $34(21.5)$ & $5(8.9)$ & \\
\hline TGA & $30(19.0)$ & $26(46.4)$ & \\
\hline $\mathrm{TA}$ & $13(8.2)$ & $2(3.6)$ & \\
\hline TAPVR & $10(6.3)$ & $5(8.9)$ & \\
\hline TVA & $10(6.3)$ & $0(0)$ & \\
\hline PA/IVS & $7(4.4)$ & $1(1.8)$ & \\
\hline Combination of above & $6(3.8)$ & $0(0)$ & \\
\hline Maternal age, years (range) & $28.1 \pm 5.8(15-42)$ & $26.5 \pm 6.0(15-41)$ & 0.089 \\
\hline \multicolumn{4}{|l|}{ Maternal diabetes status, $n(\%)$} \\
\hline Diabetes (Type I or Type II) & $7(4.5)$ & $0(0)$ & \multirow{3}{*}{0.272} \\
\hline Gestational diabetes & $6(3.8)$ & $3(5.4)$ & \\
\hline Not diabetic & $144(91.7)$ & $53(94.6)$ & \\
\hline
\end{tabular}

Diagnoses are abbreviated: HLHS, hypoplastic left heart syndrome; TOF, tetralogy of Fallot; TGA, transposition of the great arteries; TA, truncus arteriosus; TAPVR, total anomalous pulmonary venous return; TVA, tricuspid valve atresia; PA/IVS, pulmonary valve atresia with intact ventricular septum; CCHD, critical congenital heart disease. $* p<0.05$.

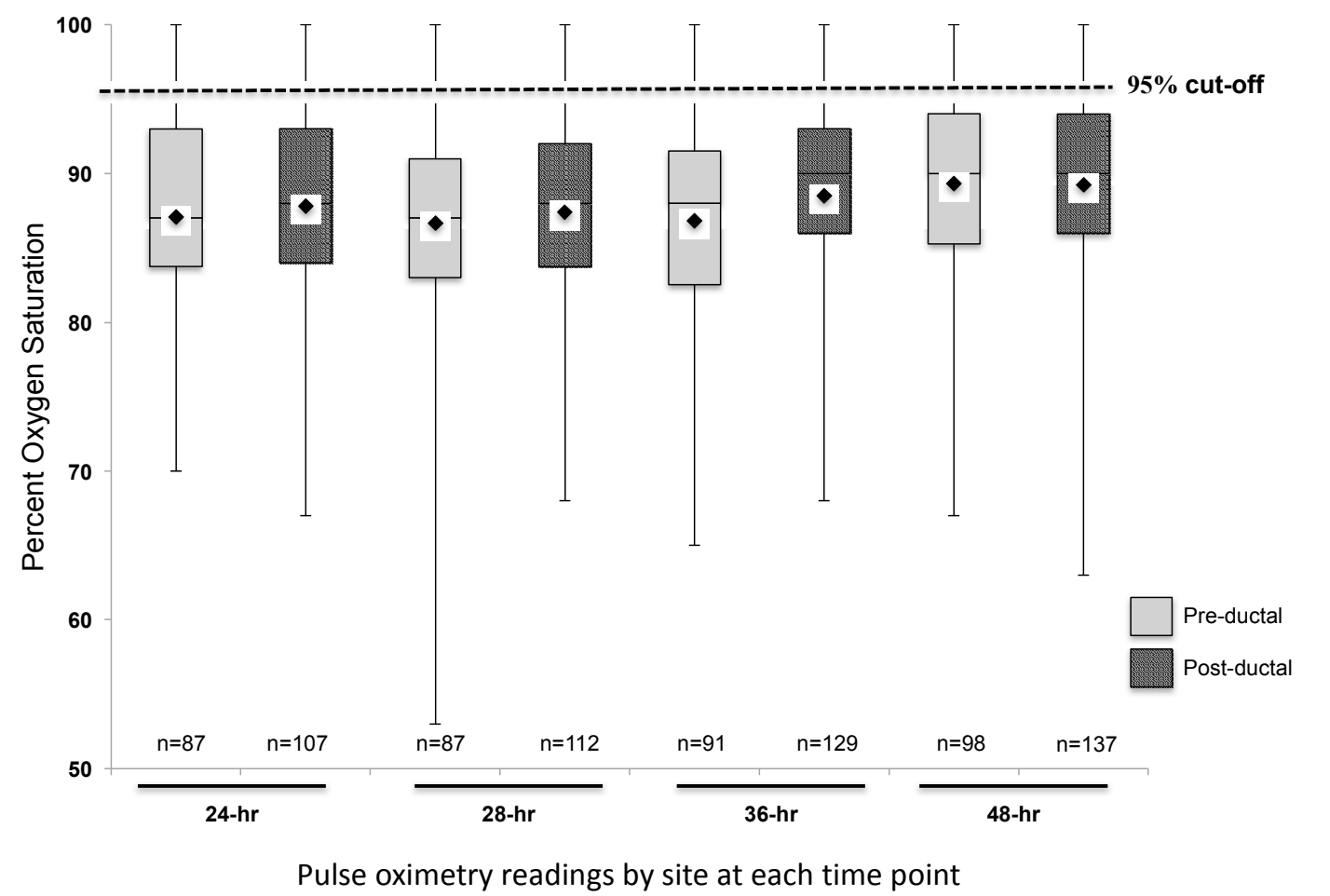

Figure 1. Pre- and post-ductal pulse oximetry data at $24,28,36$, and $48 \mathrm{~h}$ of life $(n=158)$. Mean saturation at each time point indicated by the diamond on each box plot. 

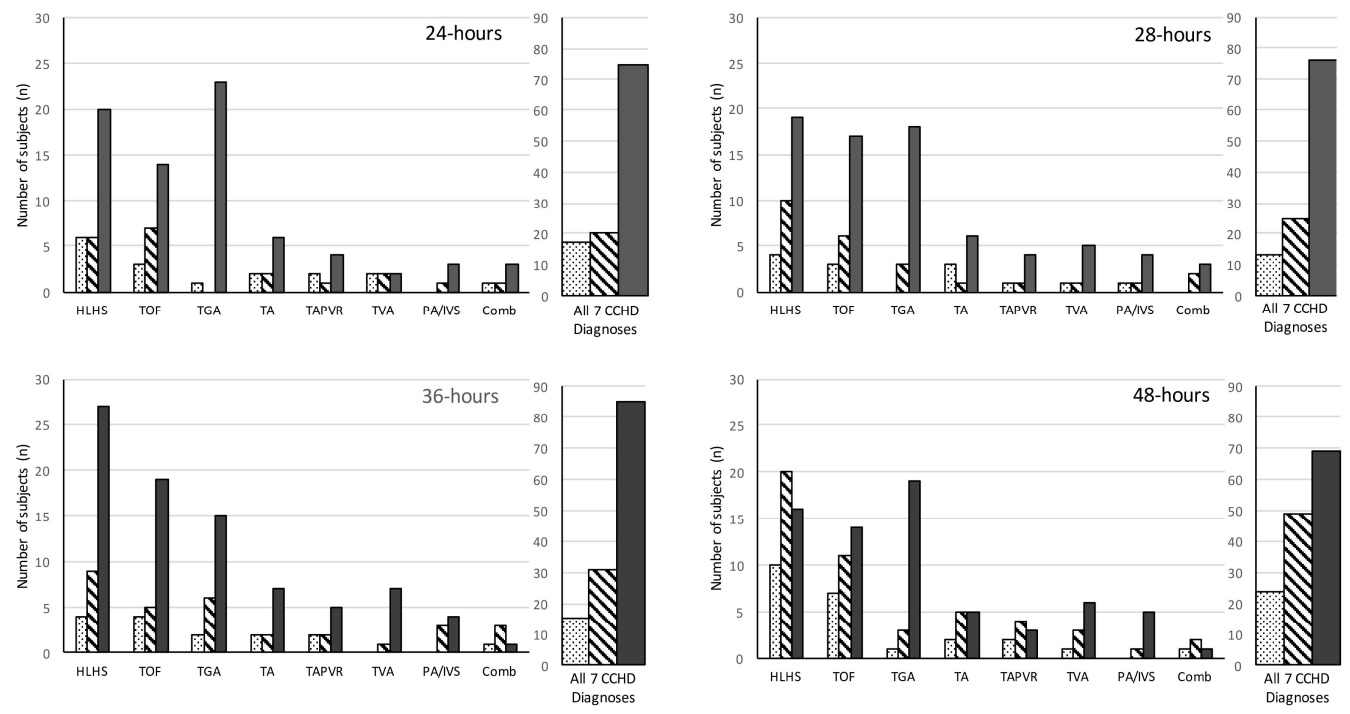

Sat $\geq 95 \%$ and $\leq 3 \%$ difference

$\mathbb{\mathbb { N }}$

Sat $90-94 \%$ and $>3 \%$ difference between pre- and post-ductal

Sat $<90 \%$ pre- or post-ductal

Figure 2. Pulse oximetry by saturation group at $24,28,36$, and $48 \mathrm{~h}$ of life $(n=158)$.

Table 2. Pre- and post-ductal pulse oximetry readings, PGE administration, and oxygen supplementation data at $24,28,36$, and $48 \mathrm{~h}$ of life $(n=158) .{ }^{*} p<0.05$.

\begin{tabular}{|c|c|c|c|c|c|}
\hline & & No Support & PGE & Oxygen & Both PGE and Oxygen \\
\hline 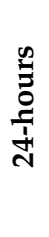 & $\begin{array}{l}\text { Pre-ductal } \\
\text { Post-ductal }\end{array}$ & $\begin{array}{c}90.3 \pm 7.7 \\
(77-100) \\
(n=7) \\
89.4 \pm 5.4 \\
(80-97) \\
(n=14)\end{array}$ & $\begin{array}{c}88.6 \pm 7.3 \\
(75-100) \\
(n=30) \\
87.8 \pm 6.3 \\
(73-99) \\
(n=33)\end{array}$ & $\begin{array}{c}86.6 \pm 4.3 \\
(80-95) \\
(n=14) \\
88.9 \pm 6.0 \\
(78-97) \\
(n=18)\end{array}$ & $\begin{array}{c}85.2 \pm 7.6 \\
(70-98) \\
(n=36) \\
86.8 \pm 6.6 \\
(67-100) \\
(n=42)\end{array}$ \\
\hline$\frac{\infty}{3}$ & $\begin{array}{l}\text { Pre-ductal } \\
\text { Post-ductal }\end{array}$ & $\begin{array}{c}88.0 \pm 5.0 \\
(81-95) \\
(n=7) \\
90.3 \pm 6.2 \\
(78-100) \\
(n=14)\end{array}$ & $\begin{array}{c}88.3 \pm 6.8 \\
(74-100) \\
(n=28) \\
88.5 \pm 7.4 \\
(70-100) \\
(n=35)\end{array}$ & $\begin{array}{c}84.6 \pm 7.1 \\
(72-96) \\
(n=18) \\
85.7 \pm 6.7 \\
(72-95) \\
(n=20)\end{array}$ & $\begin{array}{c}86.1 \pm 7.8 \\
(53-96) \\
(n=34) \\
86.2 \pm 7.6 \\
(68-100) \\
(n=43)\end{array}$ \\
\hline 号 & $\begin{array}{l}\text { Pre-ductal } \\
\text { Post-ductal }\end{array}$ & $\begin{array}{c}89.0 \pm 9.2 \\
(77-99) \\
(n=4) \\
90.0 \pm 4.9 \\
(82-100) \\
(n=15)\end{array}$ & $\begin{array}{c}88.4 \pm 5.0 \\
(76-100) \\
(n=33) \\
90.2 \pm 4.4 \\
(79-98) \\
(n=46)\end{array}$ & $\begin{array}{c}86.3 \pm 8.0 \\
(65-98) \\
(n=16) \\
88.9 \pm 7.0 \\
(72-100) \\
(n=22)\end{array}$ & $\begin{array}{c}85.5 \pm 8.9 \\
(65-100) \\
(n=38) \\
86.1 \pm 8.2 \\
(68-99) \\
(n=46)\end{array}$ \\
\hline 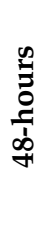 & $\begin{array}{l}\text { Pre-ductal } \\
\text { Post-ductal }\end{array}$ & $\begin{array}{c}91.0 \pm 5.3 \\
(80-97) \\
(n=8) \\
90.3 \pm 6.4 \\
(75-100) \\
(n=21)\end{array}$ & $\begin{array}{c}90.4 \pm 6.4 \\
(70-100) \\
(n=42) \\
90.5 \pm 5.2 \\
(75-99) \\
(n=51)\end{array}$ & $\begin{array}{c}89.8 \pm 4.5 \\
(81-98) \\
(n=13) \\
91.5 \pm 4.8 \\
(80-97) \\
(n=20)\end{array}$ & $\begin{array}{c}87.4 \pm 7.3 * \\
(67-100) \\
(n=35) \\
86.3 \pm 7.0 * \\
(63-100) \\
(n=45)\end{array}$ \\
\hline
\end{tabular}

\subsection{Secondary Analysis: Hypothetical Screening of Study Cohort}

Application of the AAP screening cutoffs to the retrospective data independently at each time point (not allowing for rescreens) would have resulted in $67 \%$ failing a screen at $24 \mathrm{~h}$ (75 of 112 subjects), $66.7 \%$ at $28 \mathrm{~h}$ ( 76 of 114 subjects), $64.9 \%$ at $36 \mathrm{~h}$ ( 85 of 131 subjects), and $48.6 \%$ at $48 \mathrm{~h}$ (69 of 142 subjects). A sequential screening analysis, similar to the AAP protocol, was subsequently 
applied to 149 subjects as 9 of the 158 subjects were excluded due to an inadequate number of pulse oximetry readings to apply the AAP protocol (Figure 3). Twenty-nine subjects who screened negative with passing pulse oximetry readings resulted in a $19.5 \%$ hypothetical false negative screening rate in our cohort. The 29 false negative screened subjects represented all diagnosis categories. The false negative screening rate was $18.8 \%(n=80)$ in subjects with a prenatal concern for CCHD and $20.3 \%$ $(n=69)$ in subjects without a prenatal concern for CCHD.

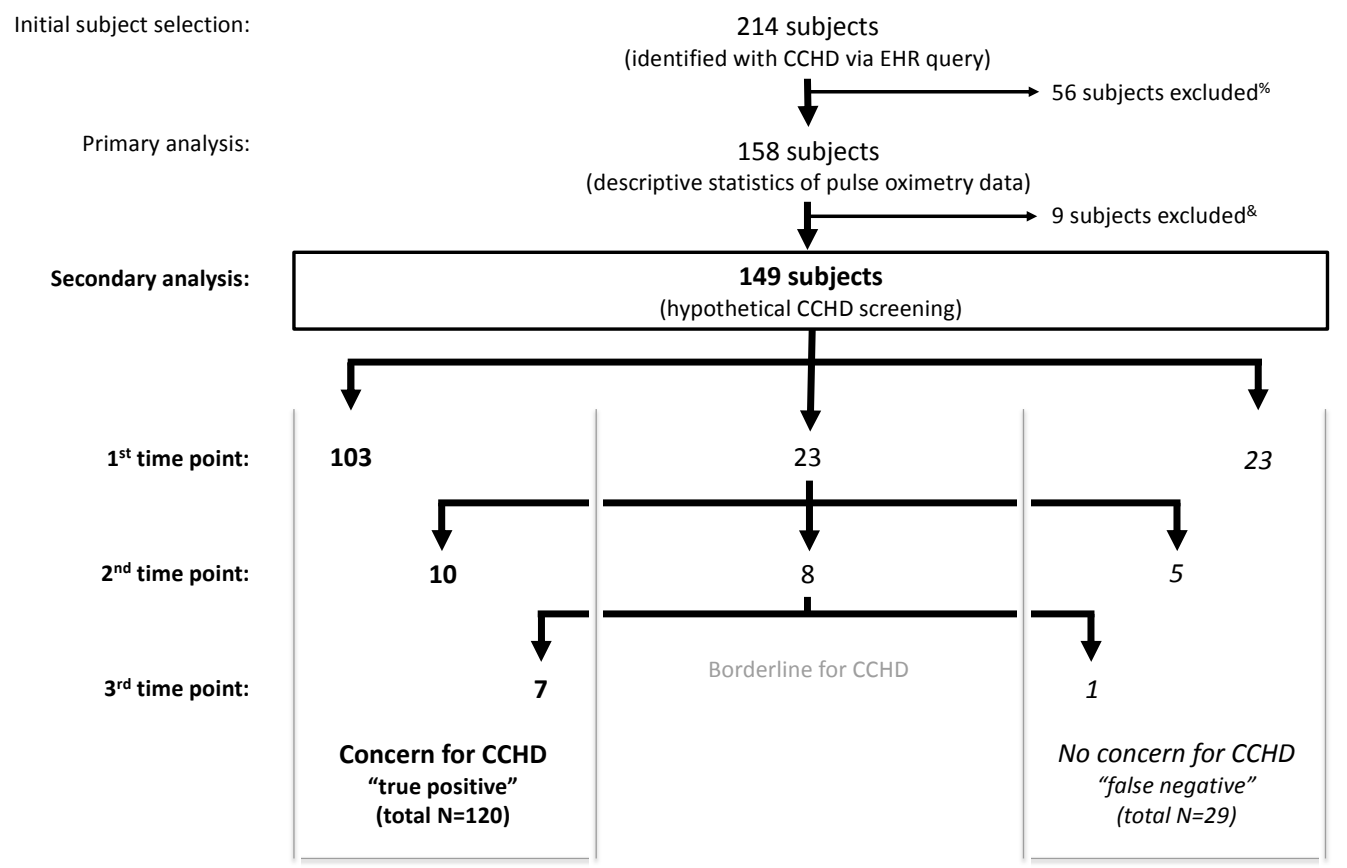

Figure 3. Application of the American Academy of Pediatrics (AAP) protocol to retrospective data in newborns with known CCHD $(n=149)$ results in 120 true positive screens and $80.5 \%$ sensitivity.

$\%$ Exclusion criteria: insufficient pulse oximetry data in the electronic health record (EHR), death prior to $24 \mathrm{~h}$ of life, atrial septostomy or surgery prior to $24 \mathrm{~h}$ of life. \& Inadequate number of pulse oximetry readings to complete the AAP protocol.

\section{Discussion}

In this study, we report mean pulse oximetry readings in a cohort of newborns with CCHD at altitude and thereby demonstrate the expected hypoxemia of the seven targeted CCHD diagnoses. Prior studies of newborn oxygen saturations have suggested a wider range and lower average saturations for infants born at higher altitude compared to those born at sea level $[3,13,15]$. We demonstrate in our cohort of infants at moderate altitude with the seven CCHD diagnoses targeted by the AAP (HLHS, TGA, TA, TAPVR, TVA, PA/IVS), that the mean pulse oximetry values are lower than recommended cutoffs for newborn screening. Wright and colleagues [3] evaluated the pulse oximetry screening protocol at 5557 feet $(1694 \mathrm{~m})$ and reported a mean pre-ductal saturation of $97.2 \%( \pm 1.9)$ and a post-ductal saturation of $97.2 \%( \pm 2.1)$ in 1003 healthy newborns at approximately $24 \mathrm{~h}$ of life ( $23.8 \pm 2.3 \mathrm{~h}$ of life, mean $\pm \mathrm{SD}$ ), similar to what would be expected in a healthy newborn cohort at sea level and significantly higher than our cohort of infants with CCHD. Another study performed at a similar altitude demonstrated lower mean saturations of $92 \%$ and $93 \%$, at 24 and $48 \mathrm{~h}$, respectively [12]. Additionally, despite similar average saturation measurements, Wright et al. demonstrated a higher rate of screen failure. This variability certainly supports the need for further study of oxygen saturation and the screening protocol at moderate altitude. 
In a secondary analysis we evaluated the hypothetical effectiveness of a sequential pulse oximetry screening protocol similar to the AAP-recommended protocol by application in a population of infants with known CCHD. By evaluating a cohort with known CCHD, we were able to demonstrate a $19.5 \%$ false negative screening rate, or a true positive screen capture rate of $80.5 \%$, at moderate altitude. Ailes and colleagues sought to address the difficulties in studying this rare disease by simulation of the population [17] and we present an alternative strategy that yields comparable findings. Our findings are comparable to the true positive screening rate found by Thangaratinam and colleagues [18], who performed a meta-analysis in 2012 of 13 studies at sea level evaluating pulse oximetry screening and calculated an overall sensitivity of $76.5 \%$ in screening for CCHD. Further, our retrospective evaluation revealed a similar sensitivity to that of two other large prospective studies $(65.5 \%$ and $75 \%)[19,20]$. Notably, the retrospective study design allowed us to perform this evaluation in a relatively large cohort of patients with CCHD that has not yet been achievable with a prospective study design [18].

Evaluations of pulse oximetry screening of neonates supported in the intensive care unit have raised concerns for increased false positive rates, issues with oxygen supplementation, and the appropriate timing of screening [21-24]. Additionally, a study performed by Lueth and colleagues evaluated a modification to the AAP-recommended screening protocol with transient oxygen supplementation in newborns at moderate altitude. Their intent was to replicate sea-level atmospheric oxygen tension and induce pulmonary vasodilatation to potentiate the neonatal transition [11]. This group found an increase in specificity of screening with a reduction in false positive screens by providing transient supplemental oxygen to newborns with an indeterminate or borderline first screen at moderate altitude [11]. Because low oxygen saturation in CCHD is primarily caused by shunting of de-oxygenated blood to the systemic circulation, oxygen supplementation in clinical practice does not typically normalize the percent saturation to greater than $95 \%$ in hypoxemic CCHD. It is difficult to know to what degree oxygen supplementation resulted in an increase in pulse oximetry reading in our study; however, we found no difference in mean oxygen saturation between patients with CCHD treated with supplemental oxygen versus those without supplemental oxygen. Nevertheless, Table 2 shows the range of saturations in both subjects receiving oxygen and those with no support exceeding 95\%. Of the 29 false negative subjects in our study, 15 received supplemental oxygen at one or more pulse oximetry readings (14 did not receive oxygen supplementation). It is certainly possible that removal of oxygen support in the subjects in our study with CCHD would induce further hypoxemia and increase the specificity of the screening test, however, we are unable to make this assessment from the data gathered. This question requires further investigation and the effect of oxygen supplementation on the screening protocol remains unclear. Our mean saturation data do provide some support to the findings of Lueth and colleagues suggesting that oxygen supplementation may not have a negative impact on the sensitivity of pulse oximetry screening for CCHD in newborns. We would not advocate for screening of hypoxemic newborns on oxygen supplementation in the intensive care unit at this time as this is not the intent of the screening guideline [6], however, the findings of this study may inform discussion regarding the timing and process for screening of well infants born at moderate-high altitude who frequently require supplemental oxygen therapy in the first days and weeks of life [13,25]. Certainly, any consideration of pulse oximetry screening for CCHD in the context of supplemental oxygen should be taken with scrutiny both of the patient physiology and use of the screening protocol guideline.

Our retrospective application of the AAP screening protocol did not allow us to exactly replicate the timing of the AAP screening protocol (three screens separated by $1 \mathrm{~h}$ each). In addition, the AAP screening protocol is meant to evaluate asymptomatic newborns not presenting in the first $24 \mathrm{~h}$ of life, whereas we studied newborns diagnosed with known CCHD. We identify both of these as limitations to this study, however, we used this cohort of infants with known CCHD to estimate the sensitivity of the screening protocol. Further, our retrospective approach resulted in 158 subjects while the very large prospective studies yielded 24-29 subjects with congenital heart disease after screening 20,000-40,000 
newborns [18]. Certainly, a future direction will be to apply this study design to a larger cohort of infants with CCHD. An additional limitation is that we found a difference in CCHD diagnoses between the subjects included in the study when compared to those excluded. A disproportionate number of the subjects with transposition of the great arteries were excluded because of atrial septostomy performed before $24 \mathrm{~h}$ of life (commonly required for infants with TGA). This may suggest that our cohort of subjects is not representative of the general population of newborns with CCHD, however, newborns requiring atrial septostomy early in life are more likely to present in extremis and not require pulse oximetry screening to identify disease. Finally, this retrospective assessment was dependent on the EHR with pulse oximetry readings that were subject to measurement and recording error from the bedside care provider. These values were not taken for formal screening or research purposes, rather, the readings were collected as a part of routine clinical care. However, these subjects all received hourly oximetry readings recorded in the EHR and the standard is for validation and confirmation by a bedside nurse with interpretation of the waveform when the infants were calm. We identify this limitation to the study design and that of measurement and documentation error by the clinical care team in the EHR. Finally, we applied the screening protocol to newborns who received PGE, oxygen, or both PGE and oxygen, to allow comparison with those who did not receive either PGE or oxygen. We recognize the contribution of both PGE and supplemental oxygen in the interpretation of subjects' pulse oximetry data; however, our results suggest that even when oxygen and PGE were administered the infants still had pulse oximetry values that were low and the therapy did not change the outcome of screening with pulse oximetry in these newborns.

We present the first report evaluating the oxygen saturations of a cohort of infants with known CCHD at moderate altitude. Additionally, we present the results of a hypothetical AAP-recommended pulse oximetry screening protocol in this same cohort. We also found that oxygen supplementation did not impact the ability of the screening protocol to identify CCHD in this cohort, which adds to the consideration of screening in neonatal intensive care units and at higher altitudes. We cannot assess the false positive rate of screening at moderate altitude with our cohort and encourage continued efforts for large population-based studies to evaluate pulse oximetry screening for CCHD.

Author Contributions: J.S.K. designed the study, performed data extraction, interpreted data, and was the primary author of the manuscript. M.W.A. performed data extraction, statistical analysis, and revised the manuscript. M.K.S. was the secondary mentor on the project, interpreted data, and revised the manuscript. C.M.R. was the primary mentor on the project, designed the study, interpreted data, and revised the manuscript. All authors approved the final manuscript as submitted and agree to be accountable for all aspects of work.

Funding: This research received no external funding.

Conflicts of Interest: Sontag has received grants from the National Institutes of Heart Lung and Blood Disorders, the Health Services and Resources Administration, and the Cystic Fibrosis Foundation. Kim, Ariefdjohan, and Rausch declare that they have no conflicts of interest.

\section{References}

1. Hoffman, J.I.E. It Is Time for Routine Neonatal Screening by Pulse Oximetry. Neonatology 2011, 99, 1-9. [CrossRef] [PubMed]

2. Wren, C.; Richmond, S.; Donaldson, L. Presentation of Congenital Heart Disease in Infancy: Implications for Routine Examination. Arch. Dis. Child. Fetal Neonatal Ed. 1999, 80, F49-F53. [CrossRef] [PubMed]

3. Wright, J.; Kohn, M.; Niermeyer, S.; Rausch, C.M. Feasibility of Critical Congenital Heart Disease Newborn Screening at Moderate Altitude. Pediatrics 2014, 133, e561-e569. [CrossRef] [PubMed]

4. Peterson, C.; Grosse, S.D.; Oster, M.E.; Olney, R.S.; Cassell, C.H. Cost-Effectiveness of Routine Screening for Critical Congenital Heart Disease in US Newborns. Pediatrics 2013, 132, e595-e603. [CrossRef] [PubMed]

5. Mahle, W.T.; Newburger, J.W.; Matherne, G.P.; Smith, F.C.; Hoke, T.R.; Koppel, R.; Gidding, S.S.; Beekman, R.H.; Grosse, S.D. Role of Pulse Oximetry in Examining Newborns for Congenital Heart Disease: A Scientific Statement from the AHA and AAP. Pediatrics 2009, 124, 823-836. [CrossRef] [PubMed] 
6. Mahle, W.T.; Martin, G.R.; Beekman, R.H.; Morrow, W.R. Section on Cardiology and Cardiac Surgery Executive Committee. Endorsement of Health and Human Services Recommendation for Pulse Oximetry Screening for Critical Congenital Heart Disease. Pediatrics 2012, 129, 190-192. [PubMed]

7. Martin, G.R.; Beekman, R.H.; Mikula, E.B.; Fasules, J.; Garg, L.F.; Kemper, A.R.; Morrow, W.R.; Pearson, G.D.; Mahle, W.T. Implementing Recommended Screening for Critical Congenital Heart Disease. Pediatrics 2013, 132, e185-e192. [CrossRef] [PubMed]

8. Kemper, A.R.; Mahle, W.T.; Martin, G.R.; Cooley, W.C.; Kumar, P.; Morrow, W.R.; Kelm, K.; Pearson, G.D.; Glidewell, J.; Grosse, S.D.; et al. Strategies for Implementing Screening for Critical Congenital Heart Disease. Pediatrics 2011, 128, e1259-e1267. [CrossRef] [PubMed]

9. Plana, M.N.; Zamora, J.; Suresh, G.; Fernandez-Pineda, L.; Thangaratinam, S.; Ewer, A.K. Pulse Oximetry Screening for Critical Congenital Heart Defects. Cochrane Database Syst. Rev. 2018, 3, CD011912. [CrossRef] [PubMed]

10. Oster, M.E.; Aucott, S.W.; Glidewell, J.; Hackell, J.; Kochilas, L.; Martin, G.R.; Phillippi, J.; Pinto, N.M.; Saarinen, A.; Sontag, M.; et al. Lessons Learned from Newborn Screening for Critical Congenital Heart Defects. Pediatrics 2016, 137, e20154573. [CrossRef] [PubMed]

11. Lueth, E.; Russell, L.; Wright, J.; Duster, M.; Kohn, M.; Miller, J.; Eller, C.; Sontag, M.; Rausch, C. A Novel Approach to Critical Congenital Heart Disease (CCHD) Screening at Moderate Altitude. IJNS 2016, 2, 4-11. [CrossRef]

12. Thilo, E.H.; Park-Moore, B.; Berman, E.R.; Carson, B.S. Oxygen Saturation by Pulse Oximetry in Healthy Infants at an Altitude of 1610 M (5280 Ft). What Is Normal? Am. J. Dis. Child. 1991, 145, 1137-1140. [CrossRef] [PubMed]

13. Ravert, P.; Detwiler, T.L.; Dickinson, J.K. Mean Oxygen Saturation in Well Neonates at Altitudes between 4498 and 8150 Feet. Adv. Neonatal Care 2011, 11, 412-417. [CrossRef] [PubMed]

14. Bakr, A.F.; Habib, H.S. Normal Values of Pulse Oximetry in Newborns at High Altitude. J. Trop. Pediatr. 2005, 51, 170-173. [CrossRef] [PubMed]

15. Samuel, T.Y.; Bromiker, R.; Mimouni, F.B.; Picard, E.; Lahav, S.; Mandel, D.; Goldberg, S. Newborn Oxygen Saturation at Mild Altitude Versus Sea Level: Implications for Neonatal Screening for Critical Congenital Heart Disease. Acta Paediatr. 2013, 102, 379-384. [CrossRef] [PubMed]

16. Harris, P.A.; Taylor, R.; Thielke, R.; Payne, J.; Gonzalez, N.; Conde, J.G. Research Electronic Data Capture (REDCap)_A Metadata-Driven Methodology and Workflow Process for Providing Translational Research Informatics Support. J. Biomed. Inform. 2009, 42, 377-381. [CrossRef] [PubMed]

17. Ailes, E.C.; Gilboa, S.M.; Honein, M.A.; Oster, M.E. Estimated Number of Infants Detected and Missed by Critical Congenital Heart Defect Screening. Pediatrics 2015, 135, 1000-1008. [CrossRef] [PubMed]

18. Thangaratinam, S.; Brown, K.; Zamora, J.; Khan, K.S.; Ewer, A.K. Pulse Oximetry Screening for Critical Congenital Heart Defects in Asymptomatic Newborn Babies: A Systematic Review and Meta-Analysis. Lancet 2012, 379, 2459-2464. [CrossRef]

19. De-Wahl Granelli, A.; Wennergren, M.; Sandberg, K.; Mellander, M.; Bejlum, C.; Inganäs, L.; Eriksson, M.; Segerdahl, N.; Agren, A.; Ekman-Joelsson, B.-M.; et al. Impact of Pulse Oximetry Screening on the Detection of Duct Dependent Congenital Heart Disease: A Swedish Prospective Screening Study in 39,821 Newborns. BMJ 2009, 338, a3037. [CrossRef] [PubMed]

20. Ewer, A.K.; Middleton, L.J.; Furmston, A.T.; Bhoyar, A.; Daniels, J.P.; Thangaratinam, S.; Deeks, J.J.; Khan, K.S. PulseOx Study Group. Pulse Oximetry Screening for Congenital Heart Defects in Newborn Infants (PulseOx): A Test Accuracy Study. Lancet 2011, 378, 785-794. [CrossRef]

21. Manja, V.; Mathew, B.; Carrion, V.; Lakshminrusimha, S. Critical Congenital Heart Disease Screening by Pulse Oximetry in a Neonatal Intensive Care Unit. J. Perinatol. 2015, 35, 67-71. [CrossRef] [PubMed]

22. Hu, X.-J.; Zhao, Q.-M.; Ma, X.-J.; Yan, W.-L.; Ge, X.-L.; Jia, B.; Liu, F.; Wu, L.; Ye, M.; Huang, G.-Y. Pulse Oximetry Could Significantly Enhance the Early Detection of Critical Congenital Heart Disease in Neonatal Intensive Care Units. Acta Paediatr. 2016, 105, e499-e505. [CrossRef] [PubMed]

23. Goetz, E.M.; Magnuson, K.M.; Eickhoff, J.C.; Porte, M.A.; Hokanson, J.S. Pulse Oximetry Screening for Critical Congenital Heart Disease in the Neonatal Intensive Care Unit. J. Perinatol. 2016, 36, 52-56. [CrossRef] [PubMed] 
24. Van Naarden Braun, K.; Grazel, R.; Koppel, R.; Lakshminrusimha, S.; Lohr, J.; Kumar, P.; Govindaswami, B.; Giuliano, M.; Cohen, M.; Spillane, N.; et al. Evaluation of Critical Congenital Heart Defects Screening Using Pulse Oximetry in the Neonatal Intensive Care Unit. J. Perinatol. 2017, 37, 1117-1123. [CrossRef] [PubMed]

25. Niermeyer, S.; Shaffer, E.M.; Thilo, E.; Corbin, C.; Moore, L.G. Arterial Oxygenation and Pulmonary Arterial Pressure in Healthy Neonates and Infants at High Altitude. J. Pediatr. 1993, 123, 767-772. [CrossRef]

(C) 2018 by the authors. Licensee MDPI, Basel, Switzerland. This article is an open access article distributed under the terms and conditions of the Creative Commons Attribution (CC BY) license (http:/ / creativecommons.org/licenses/by/4.0/). 